\title{
Projectile charge-state dependence of $K$-shell ionization by silicon ions: A comparison of Coulomb ionization theories for direct ionization and electron capture with $\mathrm{x}$-ray production data
}

\author{
F. D. McDaniel, J. L. Duggan, and George Basbas \\ Department of Physics, North Texas State University, * Denton, Texas 76203 \\ P. D. Miller \\ Oak Ridge National Laboratory, ${ }^{\dagger}$ Oak Ridge, Tennessee 37830 \\ Grzegorz Lapicki \\ Department of Physics, New York University, ${ }^{\ddagger}$ New York, New York 10003 \\ (Received 26 April 1977)
}

\begin{abstract}
$\mathrm{X}$-ray-production cross sections measured in $\mathrm{K}$-shell ionization of ${ }_{21} \mathrm{Sc},{ }_{22} \mathrm{Ti},{ }_{29} \mathrm{Cu}$, and ${ }_{32} \mathrm{Ge}$ by $52-\mathrm{MeV}$ ${ }_{14}^{28} \mathrm{Si}^{+q}$ projectiles with $q=7$ to 14 are reported, which demonstrate, through their charge-state dependence, the validity of a recently developed electron-capture theory with a reduced binding effect. Furthermore, the data provide evidence for the applicability of the perturbed stationary-state theory of direct ionization for values of $0.44 \leq Z_{1} / Z_{2} \leq 0.67$.
\end{abstract}

\section{INTRODUCTION}

$\mathrm{X}$-ray production data ${ }^{1}$ are in agreement with the predictions of the direct ionization theory ${ }^{2,3}$ for projectile $\left(Z_{1}\right)$-target $\left(Z_{2}\right)$ combinations with atomic numbers such that $Z_{1} / Z_{2} \ll 1$. This theory was developed in the perturbed-stationary state (binding $^{2}$ and polarization ${ }^{3}$ effects) approach with the inclusion of a Coulomb deflection effect and resulted in predictions superior to those of the plane-wave Born approximation ${ }^{4}$ (PWBA). However, just as in the PWBA, this theory considers only direct ionization to the continuum of the target atom and is independent of the projectile charge state. We report data which demonstrate simultaneously the validity of the direct ionization theory for $Z_{1} / Z_{2} \lesssim 1$ and that electron capture should be treated in the manner described by a recently developed theory. ${ }^{5}$ Furthermore, a modification of this treatment of electron capture is proposed that results in calculated cross sections which improve the agreement with the measured values.

We have measured $K$-shell $\mathrm{x}$-ray production cross sections for thin solid targets with atomic number $Z_{2}=21,22,29$, and 32 for $52-\mathrm{MeV}{ }_{14}^{28} \mathrm{Si}^{+\alpha}$ projectiles with incident charge states $q=7-14$. A dramatic increase in the target cross sections for projectiles with $K$-shell vacancies is attributed to electron capture to the $K^{\prime}$ shell of the projectile. ${ }^{6}$ Only a qualitative insight into this process is gained by an empirical scaling ${ }^{7}$ of the Oppenheim$\mathrm{er}^{8}$-Brinkman-Kramers ${ }^{9}$ (OBK) electron-capture cross sections. The cross sections of Nikolaev, ${ }^{10}$ calculated with screened hydrogenic wave functions and observed binding energies in the OBK approximation, also require empirical scaling. ${ }^{6,11,12}$

\section{EXPERIMENTAL PROCEDURE}

Silicon ions were obtained from the Tandem Van de Graaff accelerator at Oak Ridge National Laboratory. The high incident charge states were produced with $\mathrm{N}_{2}$ gas or carbon foil strippers placed in the beam line and selected by magnetic analysis. Target $\mathrm{x}$-ray emission was detected in a $\mathrm{Si}(\mathrm{Li})$ spectrometer having a resolution of $175 \mathrm{eV}$ at $5.94 \mathrm{keV}$. Details of the experimental arrangement and data analysis are discussed elsewhere. ${ }^{1,13}$

\section{RESULTS AND DISCUSSION}

Figure 1 shows the measured values of effective cross sections $\bar{\sigma}_{K X}$, comparable to cross sections that would be obtained from averages over thickness ${ }^{12}$ weighted by the fractions of the ${ }^{28} \mathrm{Si}$ beam with zero, one, or two $K$ vacancies that are created as the ion traverses the target foil. These cross sections are essentially independent of incident charge state for $q=7-11$. For vanishing thin targets they are enhanced by a factor of about 5 for fully stripped ions $(q=14)$ and by a factor of more than 2 for ions with one $K$-shell electron $(q=13)$. These increases are due to electron capture from the $K$ shell of the target to the $K^{\prime}$ shell of the projectile. A small enhancement in $\bar{\sigma}_{K X}$ for $q=12$ is observed, but only a negligible fraction of it $(<3 \%)$ could be explained by electron capture to an unoccupied state in the $L^{\prime}$ shell of the projectile. ${ }^{5}$ However, a metastable state with a $K^{\prime}$-shell vacancy in the incident, He-like, Si 


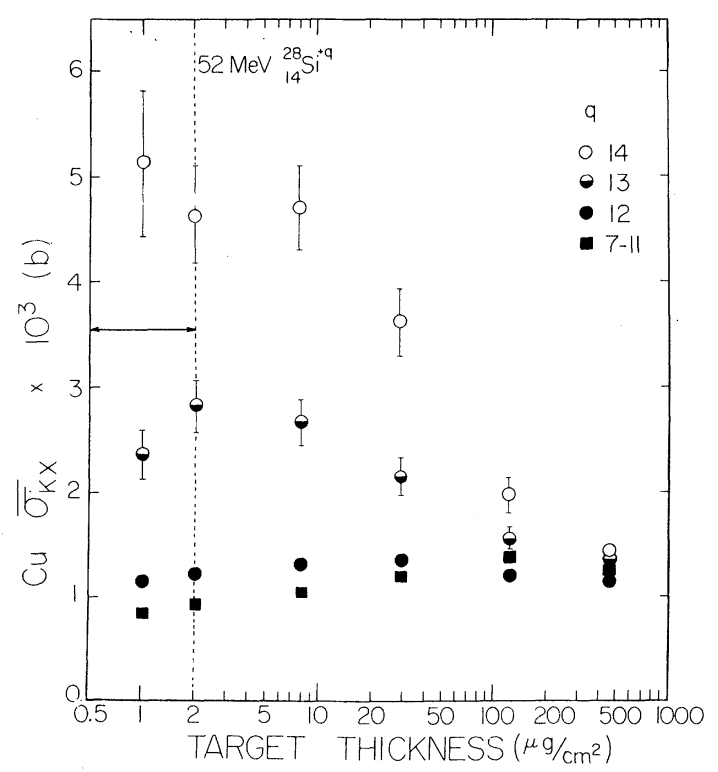

FIG. 1. Effective cross sections $\bar{\sigma}_{K X}$ for target $K$ shell $x$-ray production by projectiles in different charge states $q$ as a function of copper thickness. The doubleended arrow denotes the range of the data whose arithmetic average was taken to represent the cross sections for vanishingly thin targets. For $q=7-11$, the averages of the data listed in Table I are plotted.

beam may be formed as it passes through the foil stripper. ${ }^{14}$ Electron capture can account for the enhancement in $\bar{\sigma}_{K X}$ for $q=12$ if approximately $10 \%$ of the projectiles in the ion beam carry a $K$ -

TABLE I. Target $K$-shell $\mathrm{x}$-ray cross sections.

\begin{tabular}{ccccc}
\hline & ${ }_{21} \mathrm{Sc}^{\mathrm{a}}$ & ${ }_{22} \mathrm{Ti}$ & ${ }_{29} \mathrm{Cu}$ & ${ }_{32} \mathrm{Ge}$ \\
\hline$Z_{1} / Z_{2}$ & 0.667 & 0.636 & 0.483 & 0.438 \\
$\omega_{0}{ }^{\mathrm{b}}$ & 0.190 & 0.221 & 0.443 & 0.554 \\
$\omega^{\mathrm{c}}$ & 0.238 & 0.279 & 0.510 & 0.615 \\
$\rho t^{\mathrm{d}}$ & $\leq 2$ & $\leq 4$ & $\leq 2$ & $\leq 13$ \\
\hline$q$ & $\sigma_{K X} \mathrm{e}$ & $\sigma_{K X} \mathrm{e}$ & $\sigma_{K X}{ }^{\mathrm{e}}$ & $\sigma_{K X} \mathrm{e}^{\mathrm{e}}$ \\
\hline 7 & $30 \pm 4(3)$ & $14 \pm 2(3)$ & $94 \pm 10(1)$ & $30 \pm 3(1)$ \\
8 & & & $98 \pm 10(1)$ & $30 \pm 3(1)$ \\
9 & & & $85 \pm 10(1)$ & $29 \pm 3(1)$ \\
10 & & $13 \pm 2(3)$ & $96 \pm 10(1)$ & $28 \pm 3(1)$ \\
11 & & & $79 \pm 10(1)$ & $29 \pm 3(1)$ \\
12 & & $36 \pm 4(3)$ & $12 \pm 1(2)$ & $37 \pm 4(1)$ \\
13 & $14 \pm 2(4)$ & $94 \pm 9(3)$ & $26 \pm 2(2)$ & $60 \pm 8(1)$ \\
14 & $31 \pm 6(4)$ & $20 \pm 3(4)$ & $49 \pm 5(2)$ & $86 \pm 13(1)$ \\
\hline \hline
\end{tabular}

${ }^{\text {a }}$ Cross sections for ${ }_{21} \mathrm{Sc}$ obtained at an incident projectile energy of $50.4 \mathrm{MeV}$ were renormalized to $52 \mathrm{MeV}$ with the factor 1.15 determined from the ratio of theoretical cross sections.

${ }^{\mathrm{b}}$ Fluorescence yields from Bambynek et al. (Ref. 17).

${ }^{c}$ Fluorescence yields corrected for multiple ionization.

${ }^{\mathrm{d}}$ Target thickness in $\mu \mathrm{g} / \mathrm{cm}^{2}$.

e The notation $(n)$ means $10^{n} \mathrm{~b}$.

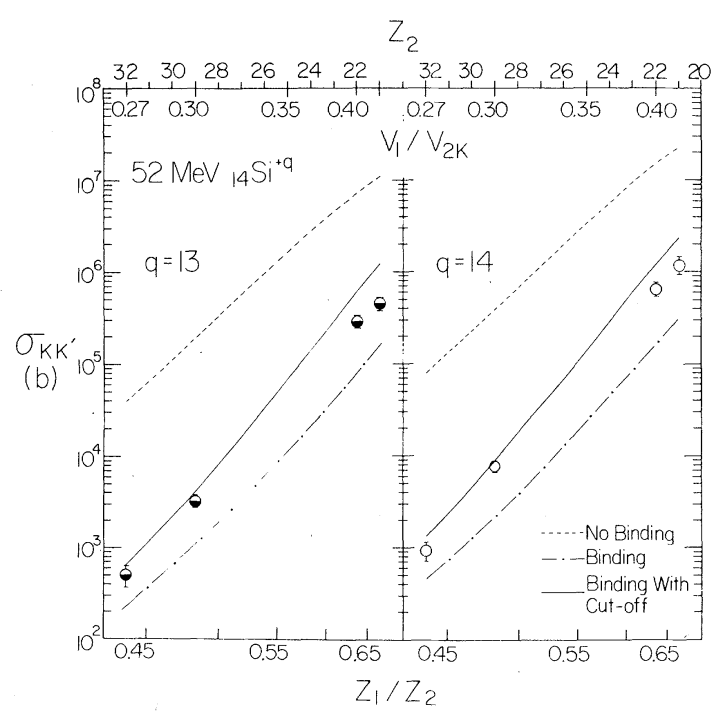

FIG. 2. Cross sections for electron capture from the $K$ shell of the target to the $K^{\prime}$ shell of silicon. The plotted values were obtained from the ionization cross sections by subtracting the data for $q=7$ from values for $q=13$ and 14 . See text for discussion.

shell vacancy into the collision.

The $\mathrm{x}$-ray production cross sections $\sigma_{K X}$ presented in Table I were obtained for targets thin enough that the charge dependence of the $x$-ray yields have been minimized. Ionization cross sections were calculated by dividing these values by the fluorescence yields corrected for multiple ionization, $\omega$. The corrections are based on vacancy distributions inferred from measured $\mathrm{x}$-ray energy shifts and the procedure of Larkins. ${ }^{15}$

For the projectile velocities $v_{1}$ reached in the present experiment, $0.27 \leqslant v_{1} / v_{2 K} \leqslant 0.42\left(v_{2 K}\right.$ is the orbital velocity of the $K$-shell electron), the low-velocity electron-capture cross sections, which include binding and Coulomb deflection effects, apply. ${ }^{5}$ In this theory, the binding effect is obtained from an integration over all impact parameters. As $v_{1} / v_{2 K}$ increases and approaches 1 , such a procedure leads to an overestimation of the binding effect. The binding should be appreciable only when the projectile is within the $K$ shell of the target; thus, as is assumed in the direct ionization theory, ${ }^{3}$ we assume that there is no increase in binding for projectiles at impact parameters larger than a prescribed cutoff radius. We have chosen the cutoff to be the expectation value of the radial distance in the $K$ shell, which is equal to $\frac{3}{2}$ of the $K$-shell radius.

In Fig. 2, electron-capture cross sections are plotted as a function of $Z_{1} / Z_{2}, Z_{2}$, and $v_{1} / v_{2 K}$. The Nikolaev OBK calculations ${ }^{10}$ with no binding effect, represented by the dashed curve, are seen 


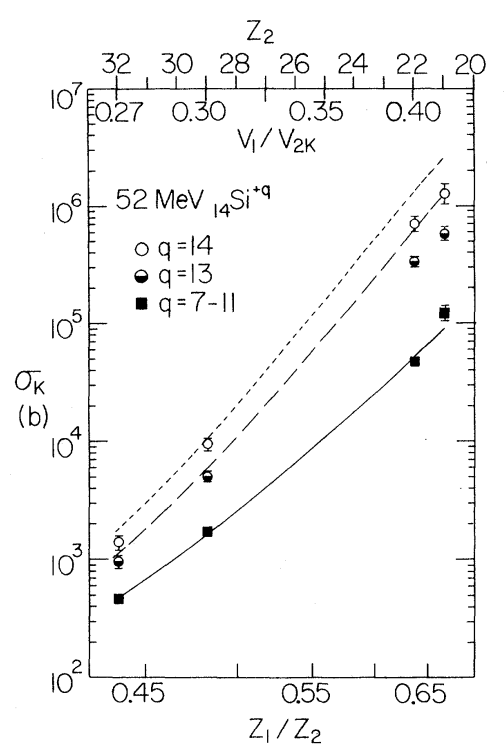

FIG. 3. Target $K$-shell ionization cross sections (direct ionization plus electron capture) vs $Z_{1} / Z_{2}$ for different projectile charge states $q$. The curves are derived from the theory of direct ionization (Refs. 2 and 3 ) and electron capture (Ref. 5) with the cutoff binding effect described in the text. The solid curve is for $q \leq 12$, the broken curve is for $q=13$, and the dashed curve is for $q=14$.

to overpredict the data by almost two orders of magnitude in some cases. In contrast, the results of the recently developed theory with the full binding effect ${ }^{5}$ indicated by the dot-dash curve, are seen to underpredict the data only by factors of from 2-4 in this intermediate-velocity region $\left(v_{1} / v_{2 K} \lessgtr 1\right)$. Furthermore, this theory with the binding effect diminished by the cutoff procedure described above provides even better agreement with the electron-capture data, as shown by the solid line. Coulomb deflection is also included in the two lower curves, but its effects for these data have been found to be negligible. Screening of the projectile nucleus by its electrons is important only at velocities higher than those considered here and does not influence the projectile chargestate dependence. ${ }^{16}$

Figure 3 illustrates the success of the theories developed in Refs. 2, 3, and 5 with the proposed cutoff binding effect for electron capture. Since the contribution of electron capture to ionization is negligible $(<3 \%)$ for incident charge states $q$ $=7-11$, the lowest curve demonstrates the validity of the direct ionization theory ${ }^{2,3}$ for $0.44 \leqslant Z_{1} / Z_{2}$ $\leqslant 0.67$. The two upper curves show that the contributions of electron capture to vacancy production are well predicted by the theory in Ref. 5 with the cutoff binding effect.

Recent data ${ }^{12}$ for $60-\mathrm{MeV}_{17}^{35} \mathrm{Cl}^{+\alpha}$ ions bombarding ${ }_{29} \mathrm{Cu}$ support the conclusions reached in this paper. For $q=16$, the electron-capture cross section, $1.6 \times 10^{4} \mathrm{~b}$, inferred from these data with $\omega=0.51$, agrees with the value $1.5 \times 10^{4} \mathrm{~b}$ obtained from the electron-capture theory ${ }^{5}$ with the cutoff binding effect. In contrast, the Nikolaev OBK cross section is $1.2 \times 10^{6} \mathrm{~b}$.

In summary, this work satisfactorily accounts for the observed charge-state dependences of the cross sections. For the data presented, target thickness effects have been minimized by employing vanishingly thin targets. The data demonstrate simultaneously the validity of the perturbed stationary state theory ${ }^{2,3}$ for $0.44 \leqslant Z_{1} / Z_{2} \leqslant 0.67$, and an electron-capture theory with a cutoff binding effect.
*Work supported by the NTSU Faculty Research Fund, The Robert A. Welch Foundation, and the Research Corporation. Travel provided by Oak Ridge Associated Universities and by the Southern Regional Educational Board.

$\dagger$ Operated by Union Carbide Corp. for ERDA.

¥Work supported by ERDA.

${ }^{1}$ R. Lear and T. J. Gray, Phys. Rev. A $\underline{8}, 2469$ (1973); T. L. Criswell and T. J. Gray, ibid. 10, 1145 (1974); F. D. McDaniel, T. J. Gray, and R. K. Gardner, ibid. 11, 1607 (1975).

${ }^{2}$ W. Brandt, R. Laubert, and I. Sellin, Phys. Rev. 151, 56 (1966); G. Basbas, W. Brandt, and R. Laubert, Phys. Rev. A 7 , 783 (1973); G. Basbas, W. Brandt, and R. H. Ritchie, ibid. 7, 1971 (1973); W. Brandt and G. Lapicki, ibid. 10, $47 \overline{4}$ (1974).

${ }^{3}$ G. Basbas, W. Brandt, and R. Laubert, Bull. Am. Phys. Soc. 18,103 (1973); and to be published.

${ }^{4}$ G. S. Khandelwal, B. H. Choi, and E. Merzbacher, At.
Data 1,103 (1969).

${ }^{5}$ G. Lapicki and W. Losonsky, Phys. Rev. A $\underline{15}, 896$ (1977).

${ }^{6}$ J. R. Macdonald et al., Phys. Rev. Lett. 29, 1291 (1972); 30, 251 (1973); 31, 621 (1973); 32, 648 (1974); Phys. Rev. A $\underline{8}, 1835$ (1973); 10, $1255(1974) ; \underline{11}, 1267$

(1975); F. Hopkins et al., Phys. Rev. Lett. 35,273

(1975); H. Tawara et al., Phys. Rev. A $13, \overline{572}$ (1976).

${ }^{7}$ A. M. Halpern and J. Law, Phys. Rev. Lett. 31, 4 (1973); 31, 620(E) (1973).

${ }^{8}$ J. R. Oppenheimer, Phys. Rev. 31, 349 (1928).

${ }^{9}$ H. C. Brinkman and H. A. Kramers, Proc. Acad. Sci. (Amsterdam) 33, 973 (1930).

${ }^{10}$ V. S. Nikolaev, Zh. Eksp. Teor. Fiz. 51, 1263 (1966) [Sov. Phys.-JETP 24, 847 (1967)].

${ }^{11} \mathrm{~J}$. A. Guffey, Ph.D. thesis (Kansas State University, 1974) (unpublished).

${ }^{12}$ T. J. Gray, P. Richard, K. A. Jamison, J. M. Hall, and R. K. Gardner, Phys. Rev. A 14, 1333 (1976); T. J. 
Gray, P. Richard, R. K. Gardner, K. A. Jamison, and J. M. Hall, Proceedings of the Third International Conference on Physics of X-Ray Spectra, 1976, National Bureau of Standards, Gaithersburg, Md., p. 272 (unpublished).

${ }^{13}$ F. D. McDaniel, T. J. Gray, R. K. Gardner, G. M. Light, J. L. Duggan, H. A. Van Rinsvelt, R. D. Lear, G. H. Pepper, J. W. Nelson, and A. R. Zander, Phys. Rev. A 12, 1271 (1975).

${ }^{14}$ I. A. Sellin, Adv. At. Mol. Phys. 12, 215 (1977); B. L. Doyle, U. Schiebel, J. R. Macdonald, J. Trow, J. A. Guffey, and L. D. Ellsworth, Bull. Am. Phys.
Soc. $21,1248(1976)$

${ }^{15}$ F. P. Larkins, J. Phys. B $\underline{4}$, L29 (1971).

${ }^{16} \mathrm{G}$. Basbas, in Electronic and Atomic Collisions, Abstract of Papers of the Ninth International Conference on Physics of Electronic and Atomic Collisions, Seattle, Washington, 1975, edited by J. S. Risley and R. Gabelle (University of Washington Press, Seattle, 1975), Vol. 1, p. 502.

${ }^{17}$ W. Bambynek, B. Crasemann, R. W. Fink, H. U. Freud, H. Mark, C. D. Swift, R. E. Price, and P. V. Rao, Rev. Mod. Phys. 44, 716 (1972); 46, 853(E) (1974). 